\title{
The Perfect Storm: Difficulties in the Treatment of Early-Onset Schizophrenia in Transitional Age Youth
}

\author{
Vanessa M Schmidt* and Ravi Shankar \\ Department of Psychiatry, University of Missouri, USA
}

*Corresponding author: Vanessa M Schmidt, Department of Psychiatry, University of Missouri, 1 Hospital Drive, MO 65201, Columbia, USA, Tel: 5738841130; Fax: 5738845936; E-mail: schmidtv@health.missouri.edu

\section{Case Report \\ Volume 1 Issue 1}

Received Date: November 08, 2017

Published Date: November 17, 2017

DOI: $10.23880 / \mathrm{mhrij}-16000110$

\section{Abstract}

Background: Early-onset schizophrenia (EOS) is defined as schizophrenia symptom onset occurring prior to age 18.However, due to comorbid psychiatric conditions, substance use and the nonspecific nature of early psychosis, there is often a significant delay in accurate diagnosis and treatment. In addition, services available to those within the transitional-age community, defined as youth between the ages of 16-25, are often limited, and the interface between child and adult mental health services poorly defined. This deficit directly affects the establishment of clinical programs designed to provide early intervention for psychotic disorders.

Case Presentation: A 17-year old male presented with paranoia, delusional thought content and disorganized behavior. The patient had previously been diagnosed with substance-induced psychosis due to similar presentations in the setting of cannabis use. However, following a 4-month incarceration with no psychiatric care, the patient demonstrated significant decompensation and an increase in duration of psychotic symptoms. Although the patient had previously demonstrated positive response to oral Aripiprazole, and had a well-documented history of medication non-compliance, insurance declined coverage of transition to Abilify Maintena due to patient age. Subsequently, the patient was initiated on oral Haloperidol. Following several episodes of dystonia, Haloperidol was slowly titrated based on response and tolerability, and transitioned to Haloperidol-Decanoate after several weeks. The patient gradually demonstrated clinical improvement in mood, behavior and delusional thought. Outpatient services were limited by current legal status, as the patient had been tried as an adult under state law. Management generally available through Children's Division and the Juvenile Office were restricted. Following multiple meetings between both inpatient and outpatient providers, the patient was scheduled for Intensive Case Management Services through a local community behavioral health provider and subsequently discharged.

Discussion: This case presents the obstacles frequently encountered in the diagnosis, treatment and outpatient management of EOS, specifically those within the transitional age community. Though services remain limited, a growing 


\section{Mental Health \& Human Resilience International Journal}

awareness of this disparity has resulted in the development of multiple early-intervention programs, as well as government-funded research initiatives designed to address the needs specific to this population.

Keywords: Schizophrenia; Substance Use; Early-Onset; Transitional Age; Early-Intervention Programs; Juvenile

Abbreviations: EOS: Early-Onset Schizophrenia; CAMHS: Child and Adolescent Mental Health Services; AMHS: Adult Mental Health Services; RAISE: Recovery After an Initial Schizophrenia Episode

\section{Background/Introduction}

Over the last one hundred and fifty years, the psychiatric community has witnessed a significant shift in its understanding of the etiology and nature of psychosis. From dementia praecox to DSM-V's schizophrenia, psychotic disorders have continued to challenge mental health providers across the globe. Naturally, this challenge increases when symptom onset occurs in childhood or early adolescence, as the majority of clinical and psychosocial deterioration takes place within the first five years of illness [1]. Although the prevalence of psychotic disorders in childhood is rare, this increases throughout adolescence, with a rate of one in 500 among 18-year olds and a lifetime prevalence of $2-3 \%$ in the adult population [2]. Schizophrenia itself affects approximately $1 \%$ of the adult population worldwide [3], with one third of those diagnosed reporting onset of illness prior to age 18 [2]. However, due to the frequent comorbidity of nonpsychotic disorders, high rate of substance use, insidious onset and/or lack of clinical training, there is often a significant delay in diagnosis, which may be attributable to the nonspecific presentation of early psychosis [4].

"Early-onset schizophrenia" is defined as schizophrenia symptom onset before the age of 18 years [5]. Although diagnostic criteria remains the same for early and adultonset schizophrenia, further development of illness trajectories may aid in determining accurate initial diagnoses, and subsequently reduce misclassification [6]. In a systematic review of 35 studies involving early-onset schizophrenia (EOS), Stentebjerg-Olesen, et al. [6] noted EOS patients were characterized by substantial impairment due to a high rate of both positive and negative symptoms, disorganized behavior, and comorbid conditions, including PTSD, ADHD, disruptive disorders and substance abuse, as well as a longer duration of untreated psychosis [6]. Delay in diagnosis and treatment, as well as a high level of negative symptoms at initial presentation and follow up, has been associated with greater overall psychopathology. Therefore, accurate detection and diagnosis of schizophrenia is imperative, as early intervention is found to play a pivotal role in determining prognosis and effectiveness of long-term management.

In addition to difficulties encountered in initial diagnosis, providing services to the child and adolescent population presents a unique challenge. Historically, limitations in research have resulted in restrictions on medication management, availability of outpatient community programs and insurance coverage. Additional obstacles include frequent changes in social environment, as well as a general dependence on the reliability of the caretaker. Finally, the transition from adolescent mental health services to adult services is often inadequate and cumbersome. The term "transitional age youth" is fluid in definition, but generally refers to those ages 16-25. Despite a growing awareness of the need for specialized care within this age group, resources are often limited and the efficacy of specific psychosocial interventions has yet to be fully explored [7]. When compared to peers without a mental health diagnosis, youth ages 16-25 with a mental illness have higher rates of substance use, homelessness, criminal justice involvement, poverty, unplanned pregnancy, unemployment and lower rates of education [8]. The universal separation of child and adult mental health services also contributes to deficits in effective transitions of care. Transitions in insurance eligibility are frequently encountered as youth reach adulthood. Consequently, transitional age youth are more likely to discontinue mental health services and are often referred to the criminal justice system [8]. In an effort to address these concerns, several early intervention programs have been designed to target the specific needs of this population, including a focus on independent living-skills, social skills, educational and vocational services, as well as therapeutic groups on relevant, ageappropriate topics, such as dating, living with roommates and family supports [8]. Despite these advancements, services within the U.S. remain largely state-dependent and highly variable [1]. Provision of care to transitional age youth, and specifically those with a diagnosis of schizophrenia, is rife with obstacles, including a higher 


\section{Mental Health \& Human Resilience International Journal}

rate of side effects associated with antipsychotic use, frequent delay in diagnosis and a lack of standardized programs designed to address the shift from child to adult services [4].

\section{Objective}

The purpose of this paper is to present the case of a 17 year old African American male diagnosed with earlyonset schizophrenia, and to facilitate discussion of obstacles to care frequently encountered by transitional age youth. Finally, a review of current therapeutic and early-intervention programs will also be presented.

\section{Case Presentation}

Patient Mr. K is a 17-year old African American male who was admitted to the Child and Adolescent Inpatient Unit on a 96-hour hold filed by parents due to bizarre and disorganized behavior. He had been released from jail to the care of his parents several hours prior, following a 4month incarceration for burglary. While incarcerated, parents report law enforcement officials noted frequent bizarre, disorganized behavior, described as eating from other inmate's trays, talking to himself and displaying a general lack of ability to assess the safety of his surroundings. Due to concerns regarding safety, he was placed in solitary confinement for a large portion of his sentence. Parents indicate he had been charged as an adult under Missouri law, and as a result, were subsequently provided with little information regarding his mental health status throughout the duration of incarceration. Despite a documented history of substanceinduced psychosis and positive response to psychotropic medications, Mr. K received no psychiatric care or medication management during this 4-month period. At the time of release, he demonstrated significant paranoia and disorientation. He reported a belief that his mother was an imposter and requested to return to jail. He refused to leave the facility with his parents and was subsequently taken to a local shelter by law enforcement. Parents then filed for a 96-hour hold, and the patient was later located and transported to the psychiatric emergency department for further evaluation.

Although Mr. $\mathrm{K}$ had been managed by previous outpatient psychiatric providers, his encounters with inpatient services began approximately 8 months prior, with an initial admission for delusional thought content and paranoia in the context of cannabis use. At that time, he demonstrated rapid, positive response to his previous outpatient regimen of Abilify, Celexa and Trazodone, which he reported having recently discontinued. He was discharged with a diagnosis of Substance-Induced Psychosis and Cannabis Use Disorder. Throughout the course of the next 3 months, Mr. K presented for three outpatient follow up appointments, one evaluation by emergency psychiatric services and one additional hospitalization. The majority of encounters note noncompliance with medications, continued cannabis use and intermittent episodes of bizarre and disorganized behavior witnessed by parents. Following his second hospitalization, he was transferred to a substance abuse treatment center and shortly after, eloped from the facility. The last outpatient appointment occurred approximately 3 weeks prior to incarceration.

Differential diagnosis at the time of admission included Substance-Induced Psychotic Disorder, Schizophrenia Spectrum disorders (including schizophreniform disorder, schizoaffective disorder, schizophrenia), and Unspecified Psychotic Disorder. A Urine Drug Screen was ordered on admission, but not completed due to repeated patient refusal. All routine laboratory tests were within normal limits. Throughout early hospitalization, Mr. K continued to demonstrate significant paranoia, voicing a fear that food, medications and soap had been poisoned. Mood lability was also observed, as he would be intermittently interactive then later characterized as isolative and irritable. He demonstrated sexually inappropriate behavior throughout early admission, repeatedly exposing genitalia and making sexually inappropriate comments to female staff and peers. On several occasions, he requested discharge, stating his wife was waiting for him in the car. During episodes of increased irritability, aggression or sexually inappropriate behavior, $\mathrm{Mr}$. $\mathrm{K}$ would receive PRN Thorazine, demonstrating mild improvement following administration. As he had previously exhibited positive response to Abilify, preliminary treatment plans included a transition from oral Abilify to AbilifyMaintena. However, insurance coverage was declined due to his age, despite a history of positive response. Oral Haldol was subsequently scheduled, with a plan for transition to Haldol-Decanoate prior to discharge. Following initiation of Haldol, Mr. K exhibited mild improvement in behavior, orientation and communication. However, due to episodes of dystonia, Haldol was decreased and Cogentin was scheduled. Over the course of several weeks, slow titration of Haldol resulted in no further dystonic reaction and provided continued clinical improvement. Mr. K began to deny significant paranoia or hallucinations on a more consistent basis. Interactions with staff and peers improved, as did appropriate participation in groups and therapeutic ward activities. After reaching a targeted oral
Vanessa M Schmidt and Ravi Shankar. The Perfect Storm: Difficulties in the Treatment of Early-Onset Schizophrenia in Transitional Age Youth. Ment Health Hum Resilience Int J 2017, 1(1): 000110.
Copyright@ Vanessa M Schmidt and Ravi Shankar. 


\section{Mental Health \& Human Resilience International Journal}

dose and demonstrating sustained clinical improvement, he was transitioned to Haldol-Decanoate.

Although considered a minor within the medical community, the treatment team experienced significant difficulty in securing outpatient case management services for Mr. K, due in large part to his age (17.5 years old) and previous legal charge as an adult. Services generally available through Children's Division or the Juvenile Office, were denied. Following multiple meetings between inpatient and outpatient providers, social workers, therapists, and community liasons, the patient was scheduled for Intensive Case Management Services through a local community behavioral health provider and subsequently discharged.

\section{Discussion}

The preceding case is an all-too familiar example of obstacles frequently encountered in the diagnosis, treatment and outpatient management of EOS, specifically those within the transitional age community. As discussed previously, timely identification of EOS is problematic in itself. An indistinct prodromal period and frequent delay in diagnosis, often followed by multiple medication trials and treatment non-compliance, can present many challenges to effective management. Behavior observed during episodes of untreated psychosis often results in referral to the justice system, and frequent encounters with law enforcement. If the adolescent is tried as an adult, as was true in this case, services provided by the Juvenile Office and Children's Division are also restricted. In addition, once a diagnosis has been confirmed and treatment plan developed, the successful interface between child and adolescent mental health services (CAMHS) and adult mental health services (AMHS) is often poorly defined or absent entirely [9]. In a U.S. study assessing the availability of transitional services in 41 states, Davis and colleagues noted one-quarter of state CAMHS and one-half of state AMHS offered no transition services. States that did provide these services were significantly limited in availability, as less than $20 \%$ offered transition services at more than one site [10]. Programs such as age-specialized case management, peer support, supervised or supported housing, vocational support, independent living preparation, community treatment, dual diagnosis and wrap-around services were among the list of current interventions. Although supported/supervised housing and wrap-around approaches were offered by several states, few provided more than one type of transition service [10]. With no universal protocol to date, a lack of standardized transition policies, variations in referral criteria and the continued bifurcation of child and adult mental health services are frequently cited as crucial barriers to the development of programs for transitional-age youth [9].

Subsequently, this deficit directly affects the establishment of clinical programs designed to provide early intervention for psychotic disorders, as a large percentage of patients report onset of symptoms before the age of 25 [11]. In a study conducted by Hafner, et al. [12] 267 patients with schizophrenia were evaluated via a standardized semistructured interview for the retrospective assessment of symptom onset. Results showed initial onset of symptoms occurred at an early and steep increase throughout adolescence until age 25 , with a pronounced peak range present for both males and females, ages 15-25 and 20-29, respectively. Additional findings noted $62 \%$ of males and $47 \%$ of females reported experiencing the first signs of mental illness before the age of 25 [12], emphasizing the importance of addressing inadequacies in transitional age mental health care.

Though services remain limited, the last twenty years has demonstrated a growing awareness of this disparity, with the establishment of multiple early intervention programs and studies aimed to assess the cognitive and social needs specific to this population. Large-scale government funding has been essential in the development of such programs throughout Australia, Great Britain and Canada. In 2009, NIMH launched the Recovery after an Initial Schizophrenia Episode (RAISE) research initiative to explore methods for implementing coordinated care for first episode psychosis in the U.S. Results of this initiative served to strengthen evidence already supporting early intervention in psychosis [13]. In recent years, the U.S. government has also acknowledged the growing need to address availability of mental health care to this population. Through a Congressional appropriations bill, SAMHSA has been directed to allocate a percentage of Mental Health Block Grants to address serious mental illness, set as 5\% in 2015 [14]. In 2016, this percentage increased to $10 \%$, and must be used to address first-break psychosis, specifically [14].

In general, two primary strategies guiding early intervention have been most frequently implemented: 1) early detection and diagnosis and 2) prompt treatment before sustained psychotic symptoms occur [15]. In an effort to identify key components of these programs, several studies have evaluated program characteristics, clinical services and eligibility criteria. Recently, Addington, et al. [16] conducted a two-stage analysis of early intervention programs, implementing a systematic review of literature designed to identify key elements of 


\section{Mental Health \& Human Resilience International Journal}

these programs. Components identified were then further assessed via a Delphi Consensus method, which rated importance of these elements on a 5-point scale [16]. 32 components were subsequently identified, and later applied by White et al. for further assessment of early intervention programs within the U.S. Of the 31 programs identified, 11 targeted first episode psychosis only, 8 were designed to serve only those deemed a "clinical high risk," and 12 programs served both. Studies noted key program elements include pharmacological interventions, family psychoeducation, cognitive-behavioral treatment and vocational services [17]. Although components are often variable, White, et al. [17] also identified "new" themes of service considered to be essential by program directors, including case management, client engagement, social skills and addressing practical needs [17]. Accessibility of these services is often determined by availability of specialized, state-specific funding and restrictions inherent to the presence of eligibility criteria. The majority of programs implement age restrictions and limit eligibility based on duration of untreated psychosis [17]. Although necessary, eligibility parameters may inadvertently exclude individuals who could benefit from program services. Future program development may profit from additional research regarding the difference in efficacy of various treatment modalities between distinct stages of early psychosis. This may aid in more universally implemented eligibility criteria.

\section{Conclusion}

The primary objective of this case report is to serve as a catalyst for further discussion of obstacles frequently encountered in the management of early onset schizophrenia within transitional age youth. Although great strides have been observed throughout the mental health community, deficits in access to care and limited availability of evidence-based community programs remain. Barriers such as variability in insurance coverage of long-acting antipsychotics, restrictions to services provided by Children's Division and the Juvenile Office, and limited step-down services following acute hospitalization may also contribute to fragmented, inadequate care. However, with continued research into the validity of specific treatment models, a template for consistent early intervention program development may prove beneficial.

\section{Consent}

At the conclusion of this inpatient stay, the mother of the patient provided consent for the publication of this case report, with the understanding that information would remain anonymous and confidential.

\section{References}

1. De Maio M, Graham P, Vaughan D, Haber L, Madonick S (2015) Review of international early psychosis programmes and a model to overcome unique challenges to the treatment of early psychosis in the United States. Early Interv Psychiatry 9(1): 1-11.

2. Rey JM (2015) IACAPAP e-Textbook of Child and Adolescent Mental Health. Geneva: International Association for Child and Adolescent Psychiatry and Allied Professions.

3. Gogtay N, Vyas NS, Testa R, Wood SJ, Pantelis C (2011) Age of Onset of Schizophrenia: Perspective from Structural Neuroimaging Studies. Schizophrenia Bulletin 37(3): 504-513.

4. Schimmelmann BG, Walger $\mathrm{P}$, Schultze-Lutter $\mathrm{F}$ (2013) The Significance of At-Risk Symptoms for Psychosis in Children and Adolescents. Can J Psychiatry 58(1): 32-40.

5. Kendhari J, Ravi Shankar, Laine Young-Walker (2016) A Review of Childhood-Onset Schizophrenia. Focus 14(3): 328-332.

6. Stentebjerg-Olsen $\mathrm{M}$, Pagsberg AK, Fink-Jensen A, Correll CU, Jeppesen P (2016) Clinical Characteristics and Predictors of Outcome of SchizophreniaSpectrum Psychosis in Children and Adolescents: A Systematic Review. J Child Adolesc Psychopharmacol 26(5): 410-427.

7. Armando M, Pontillo M, Vicari S (2015) Psychosocial interventions for very early and early-onset schizophrenia: a review of treatment efficacy. Curr Opin Psychiatry 28(4): 312-323.

8. Gilmer TP, Ojeda VD, Fawley-King K, Larson B, Garcia $P$ (2012) Change in Mental Health Service Use After Offering Youth-Specific Versus Adult Programs to Transition-Age Youths. Psychiatr Serv 63(6): 592596.

9. Paul M, Street C, Wheeler N, Singh SP (2015) Transition to adult services for young people with mental health needs: A systematic review. Clin Child Psychol Psychiatry 20(3): 436-457.

10. Davis M, Geller JL, Hunt B (2006) Within-State Availability of Transition-to-Adulthood Services for
Vanessa M Schmidt and Ravi Shankar. The Perfect Storm: Difficulties in the Treatment of Early-Onset Schizophrenia in Transitional Age Youth. Ment Health Hum Resilience Int J 2017, 1(1): 000110.
Copyright@ Vanessa M Schmidt and Ravi Shankar. 
Youths with Serious Mental Health Conditions. Psychiatr Serv 57(11): 1594-1599.

11. Wilens TE, Rosenbaum JF (2013) Transitional Aged Youth: A New Frontier in Child and Adolescent Psychiatry. J Am Acad Child Adolesc Psychiatry 52(9): 887-890.

12. Hafner $\mathrm{H}$, Maurer $\mathrm{K}$, Löffler $\mathrm{W}$, Riecher-Rössler A (1993) The Influence of Age and Sex on the Onset and Early Course of Schizophrenia. Br J Psychiatr 162: 8086.

13. Heinssen RK, Amy B Goldstein, Susan T Azrin (2014) Evidence-Based Treatments for First Episode Psychosis: Components of Coordinated Specialty Care Bethesda: National Institute of Mental Health.
14. Samhsa (2016) Substance Abuse and Mental Health Services Administration.

15. Schimmelmann BG, Schultze-Lutter F (2012) Early detection and intervention of psychosis in children and adolescents: urgent need for studies. Eur Child Adolesc Psychiatry 21(5): 239-241.

16. Addington DE, McKenzie E, Norman R, Wang J, Bond GR (2013) Essential Evidence-Based Components of First-Episode Psychosis Services. Psychiatr Serv 64(5): 452-457.

17. White DA, Luther L, Bonfils KA, Salyers MP (2015) Essential components of early intervention programs for psychosis: Available intervention services in the United States. Schizophr Res 168(1-2): 79-83. 